\title{
ELASTOPLASTIC TORSION BY QUADRATIC PROGRAMMING
}

\author{
Dianne P. O'LEARY and Wei H. YANG \\ The University of Michigan, Ann Arbor, Michigan, USA \\ Received 11 April 1978 \\ Revised manuscript received 22 May 1978
}

\begin{abstract}
A finite element scheme (together with a conjugate gradient algorithm) is demonstrated to be a very effective method for analyzing gencral elastoplastic torsion of prismatic bars posed as quadratic programming problems. Solutions for bars with elliptical and Sokolovsky's oval cross-sections are presented. The solutions for the elliptical bars agree with the existing elastic and limit plastic solutions at the two extremes of the elastic-plastic range. The algorithm also reproduces accurately the Sokolovsky solution and extends it beyond its limitations.
\end{abstract}

\section{Introduction}

Elastoplastic analysis is considered to be one of the most difficult topics in the theory of plasticity [1]. Some fully plastic solutions can be obtained by an approximation known as limit analysis [2]. But in the elastic-plastic range, only a few solutions exist in the literature. Sokolovsky [1] constructed a solution by an inverse method which does not offer extension to other elastoplastic problems. Even with such an exact solution the torque related to the solution can be obtained only by numerical integration.

The emergence of large computers in the 1960's steered the development of elastoplastic analysis in a new direction. Large finite element codes have been written to compute incrementally the elastoplastic solutions [3-5]. Some codes provide solutions only at a tremendous computing cost. Some solutions are deemed unreliable because of uncontrolled round-off error accumulation after a huge number of floating point operations. Even with the good codes the expense sometimes prohibits thorough parametric analysis which requires repeated calculations of similar solutions.

Recent advances in sparse matrix computations [6] can be employed to remedy this lack of efficiency. The matrices involved in the discretization of the elastoplastic field equations have a structured sparsity pattern. This property can be exploited to increase computational efficiency.

The finite element method [7] has revolutionized the computation of elastic structure and continuum problems. It is certainly just as effective for approximating the elastoplastic analysis. Some plasticity problems arise naturally in the form of constrained variational problems. Through the finite element method these can generally be reduced to nonlinear programming problems [8]. In particular, the elastoplastic torsion problem can be formulated as a mathematical programming problem with a quadratic objective function and a set of simple bounds as constraints. This quadratic programming problem has received special attention in the fields of operations research and computer science, and efficient algorithms are being developed $[9,10]$. The algorithm in [9] is used for the solutions in this paper. 
As examples, elliptical and Sokolovsky's oval cross-sections are chosen for the torsion solutions in the elastic-plastic range. At the lower extreme of the range the computed solutions for the elliptical bars agree with the exact elastic solutions [11]. In the upper range the solutions asymptotically approach the limit analysis solutions [12]. The oval solutions, valid for the entire elastic-plastic range, agree with the exact Sokolovsky solution in its limited range.

\section{The problem}

The elastic torsion problem is governed by the equation

$$
\nabla^{2} \phi=-2 G \theta \quad \text { in } D, \quad \phi=0 \quad \text { on } \partial D,
$$

where $\phi(x, y)$ is the stress function defined in the cross-section $D$ of a prismatic bar bounded by the closed curve $\partial D, G$ is the shear modulus, and $\theta$ is the angle of twist per unit length.

The problem defined in (1) is equivalent to the variational problem of finding $\phi(x, y)$ that minimizes

$$
J(\phi)=\frac{1}{2} \iint_{D}|\nabla \phi|^{2} \mathrm{~d} A-2 G \theta \iint_{D} \phi(x, y) \mathrm{d} A
$$

and satisfies the boundary condition $\phi=0$.

The value of $|\nabla \phi|$ at each point, representing the local magnitude of the shear stress, increases with $G \theta$. Physically, the material ceases to behave elastically when $|\nabla \phi|=\sigma_{0}$, where $\sigma_{0}$ is the yield stress. An elastic perfectly plastic model that requires

$$
|\nabla \phi| \leqslant \sigma_{0}
$$

is used as a constraint to the minimization problem (2). Thus the elastoplastic torsion problem can be restated as:

$$
\text { minimize } \quad \frac{1}{2} \iint_{D}|\nabla \bar{\phi}|^{2} \mathrm{~d} A-\bar{\theta} \iint_{D} \bar{\phi} \mathrm{d} A
$$

subject to $|\nabla \bar{\phi}| \leqslant 1$,

$$
\bar{\phi}=0 \quad \text { on } \partial D,
$$

where $\bar{\theta}=2 G \theta / \sigma_{0}$ and $\bar{\phi}=\phi / \sigma_{0}$.

It is useful to note that as $\bar{\theta} \rightarrow \infty$, the problem stated in (4) approaches the limit torsion problem:

$$
\text { maximize } \iint_{D} \bar{\phi} \mathrm{d} A, \quad \bar{\phi} \in C^{0},
$$

subject to $|\nabla \bar{\phi}| \leqslant 1$,

$$
\bar{\phi}=0 \quad \text { on } \partial D \text {, }
$$


whose solution is a surface of constant slope [12] such that $|\nabla \bar{\phi}|=1$. The finite element method used in [12] exploits this property. The condition $|\nabla \bar{\phi}| \leqslant 1$ is equivalent to $|\bar{\phi}(x, y)| \leqslant$ $d(x, y, \partial D)$, where $d(x, y, \partial D)$ is the shortest distance from the point $(x, y)$ to the boundary $\partial D$ [13]. This distance can be obtained most conveniently by solving (5). The solution of (5) is used to generate the firite element mesh system given by a set of steepest descent lines. The mesh points, which divide each line into equal intervals, are connected to form a network. Each quadrilateral mesh unit is then divided into two triangles by a diagonal. A typical mesh system is shown in fig. 1. Using a linear interpolation function to approximate $\bar{\phi}(x, y)$ in each triangle, the discrete version of (4) takes the form of a standard quadratic programming problem

$$
\begin{array}{ll}
\text { minimize } & \frac{1}{2} \boldsymbol{\phi}^{\mathrm{t}} \boldsymbol{K} \boldsymbol{\phi}-\bar{\theta} \boldsymbol{c}^{\mathrm{t}} \boldsymbol{\phi}, \\
\text { subject to } & 0 \leqslant \phi_{i} \leqslant d_{i}, \quad i=1,2, \ldots n,
\end{array}
$$

where $\boldsymbol{K}$ is a symmetric positive definite matrix, $\boldsymbol{c}$ and $\boldsymbol{d}$ are constant vectors of dimension $n$ (the number of interior nodes), and $\phi_{i}$ is the value of the unknown function $\phi(x, y)$ at the $i$ th node. The lower bounds of $\phi_{i}$ are set at zero for the counter-clockwise torque.

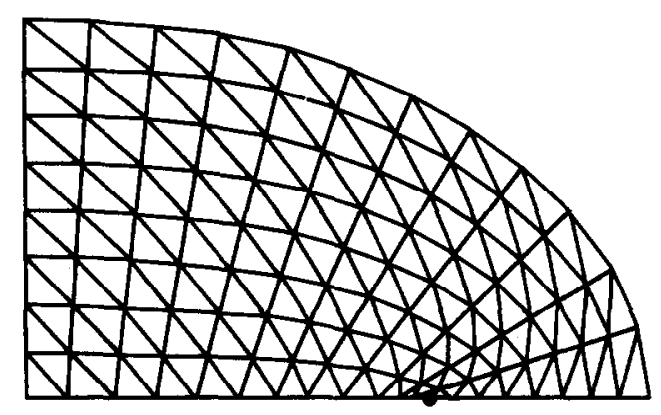

Fig. 1. A typical finite element mesh.

\section{The algorithm}

The quadratic programming problem (6) can be solved by a conjugate gradient algorithm whose basic framework is due to Polyak [14]. The solution to (6) is characterized by the conditions

$$
\left.\begin{array}{lll}
g_{i}=0 & \text { if } & 0<\phi_{i}<d_{i} \\
g_{i} \geqslant 0 & \text { if } & \phi_{i}=0 \\
g_{i} \leqslant 0 & \text { if } & \phi_{i}=d_{i}
\end{array}\right\} \quad i=1,2, \ldots n,
$$

where $\boldsymbol{g}=\boldsymbol{K} \boldsymbol{\phi}-\boldsymbol{c}$ is the gradient of $J(\phi)$. The algorithm starts from a vector $\boldsymbol{\phi}$ which satisfies the upper and lower bounds and proceeds to decrease the quadratic function at each iteration. It terminates with the optimal solution after a finite number of iterations. 
The algorithm does not modify the matrix $K$ and needs to access it only through a subroutine which forms the product of $\boldsymbol{K}$ with any given vector $\boldsymbol{p}$. Since the matrix is sparse, this is a very inexpensive operation.

The algorithm can be accelerated through the use of a positive definite matrix $M$ which has the properties that the ratio if the maximum and minimum eigenvalues of $\boldsymbol{M}^{-1} \boldsymbol{K}$ is smaller than the ratio for $\boldsymbol{K}$, and the system of equations $\overline{M g}=\boldsymbol{g}$ is easy to solve for any given vector $\boldsymbol{g}$. It is possible to take $M$ equal to the identity matrix, but a choice of $M$ equal to the diagonal of $\boldsymbol{K}$ is sometimes more effective. Other choices of $\boldsymbol{M}$ are discussed in [9].

The algorithm is as follows:

Given: an initial vector satisfying $\boldsymbol{o} \leqslant \boldsymbol{\phi} \leqslant \boldsymbol{d}$, a matrix $\boldsymbol{M}$, the initial gradient $\boldsymbol{g}=\boldsymbol{K} \boldsymbol{\phi}-\boldsymbol{c}$, and a prescribed tolerance $\epsilon$, perform:

\section{Outer iteration:}

$$
\text { 1. Set } s_{i} \leftarrow\left\{\begin{array}{cll}
-1 & \text { if } \phi_{i} \leqslant \epsilon & \text { and } g_{i}>0 \\
1 & \text { if } \phi_{i} \geqslant d_{i}-\epsilon & \text { and } g_{i}<0 \\
0 & \text { otherwise }
\end{array}\right\} \quad i=1,2, \ldots n \text {. }
$$

2. If $s$ has not changed from the previous iteration, terminate with the solution $\phi$.

3. Set $\quad \bar{g}_{i} \leftarrow g_{i} \quad$ if $s_{i}=0$

$$
g_{i} \leftarrow 0 \quad \text { otherwise } i=1,2, \ldots n \text {, }
$$

and set $\boldsymbol{g} \leftarrow-\overline{\mathbf{g}}$ and $k \leftarrow 1$.

4. Begin the inner iteration.

\section{Inner iteration:}

1. If all components of $g$ corresponding to zero components of $s$ are less than $\epsilon$ in absolute value, then start the next outer iteration.

2. Calculate a step length:

$$
\begin{aligned}
& \alpha_{o p t} \leftarrow-\boldsymbol{g}^{\mathrm{t}} \boldsymbol{p} / \boldsymbol{p}^{\mathrm{t}} \boldsymbol{K} \boldsymbol{p}, \\
& \alpha_{\max } \leftarrow \min \left[\min _{p_{i}>0}\left(d_{i}-\phi_{i}\right) / p_{i}, \min _{p_{i}<0}\left(-\phi_{i}\right) / p_{i}\right], \\
& \alpha \leftarrow \min \left(\alpha_{o p t}, \alpha_{\max }\right) .
\end{aligned}
$$

3. Update the solution and gradient:

$$
\begin{aligned}
& \boldsymbol{\phi} \leftarrow \boldsymbol{\phi}+\alpha p \\
& g \leftarrow \boldsymbol{g}+\alpha K \boldsymbol{p} .
\end{aligned}
$$


4. If $\alpha \neq \alpha_{\max }$, then proceed with step 5. Otherwise, some variable has reached a bound. Set

$$
s_{i} \leftarrow\left\{\begin{array}{cl}
-1 & \text { if } \phi_{i} \leqslant \epsilon \\
1 & \text { if } \phi_{i} \geqslant d_{i}-\epsilon
\end{array}\right\} \quad i=1,2, \ldots n
$$

If all components of $s$ are nonzero, then start the next outer iteration. Otherwise, set $k \leftarrow 1$. 5. Prepare for the next iteration; set

$$
\left.\begin{array}{ll}
\bar{g}_{i} \leftarrow\left(\boldsymbol{M}^{-1} \boldsymbol{g}\right)_{i} & \text { if } s_{i}=0 \\
\bar{g}_{i} \leftarrow 0 & \text { otherwise }
\end{array}\right\} \quad i=1,2, \ldots n .
$$

If $k=1$ then $\boldsymbol{p} \leftarrow-\overline{\boldsymbol{g}}$, else $\beta \leftarrow \boldsymbol{g}^{\mathrm{t}} \overline{\boldsymbol{g}} / \gamma(\gamma$ was computed at the previous iteration), and $\boldsymbol{p} \leftarrow-\overline{\boldsymbol{g}}+\beta \boldsymbol{p}$. Then update $\boldsymbol{\gamma} \leftarrow \boldsymbol{g}^{\mathbf{g}} \overline{\mathbf{g}}$.

Set $k \leftarrow k+1$ and start the next inner iteration.

A more complete explanation of the algorithm and proof of its convergence is given in [9]. It is advantageous to use the algorithm to find a solution with a large $\epsilon$ and then refine $\epsilon$ and restart it. This reduces the number of inner iterations. The algorithm can be implemented in less than 100 Fortran statements, plus separate subroutines for forming $\boldsymbol{K} \boldsymbol{p}$ and $\boldsymbol{M}^{-1} \boldsymbol{g}$. Notice that components of $\boldsymbol{p}$ and $\phi$ corresponding to nonzero components of $s$ do not change in the inner iterations; this should be exploited in the coding.

\section{Elliptical shaft}

The first quadrant of an ellipse with major and minor semiaxes $a$ and $b$ is shown in fig. 1 . The finite element mesh is shown as the network constructed from the steepest descent lines obtained in [12]. The value of $d(x, y, \partial D)$ at each mesh point can be easily calculated along the steepest descent line passing through the point.

With the conjugate gradient algorithm it is most convenient to start the iteration from a large value of $\bar{\theta}$ which corresponds to a nearly limit plastic solution. By decreasing $\bar{\theta}$, elastoplastic solutions with decreasing plastic zone are computed. The solution for a new $\bar{\theta}$ is obtained using the solution for the previous $\bar{\theta}$ as the initial iterate. For a fixed precision the number of iterations taken to converge to a new solution depends on the magnitude of change from the initial iterate. Thus it is natural to take large steps in $\bar{\theta}$ on the flat part of the torque vs. angle of twist curve and small steps on the steep part. Changing $\bar{\theta}$ slowly to produce more information may not necessarily incur higher computing cost. A desirable rate of decreasing $\bar{\theta}$ can be determined by experiment during computation.

When $\bar{\theta}$ is decreased to the value corresponding to the maximum elastic torque, the entire domain will become elastic $(|\nabla \phi|<1)$. Below this value the solution varies linearly with $\bar{\theta}$. There is no need to carry the computation further. 


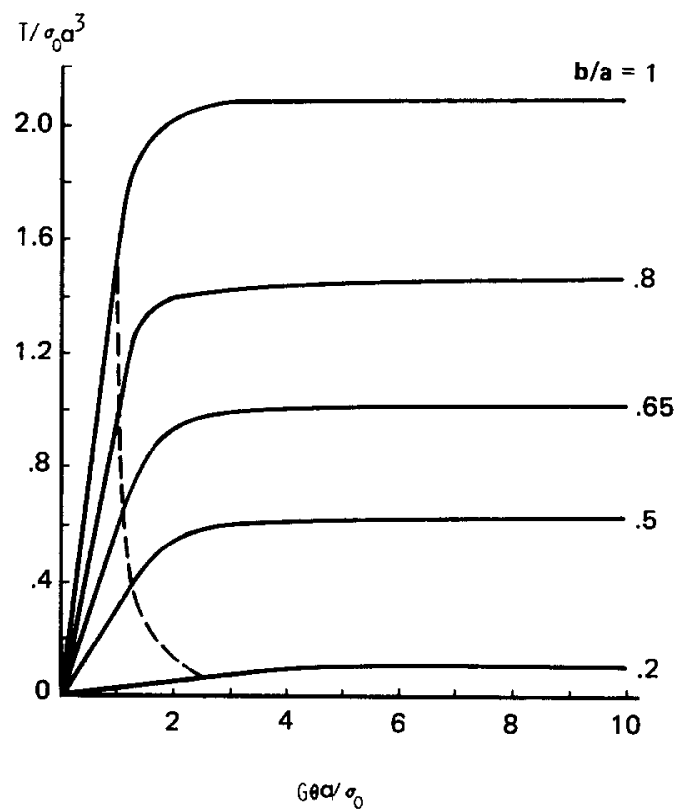

Fig. 2. Torque-angle of twist curves of elliptical shafts.

The integral $2 \iint_{D} \phi(x, y) \mathrm{d} A$ gives the torque whose dimensionless form $T /\left(\sigma_{0} a^{3}\right)$ is plotted against $\frac{1}{2} \theta a$ for various $b / a$ ratios in fig. 2 . The dotted curve separates the linear elastic solution region from the elastoplastic region. As $\bar{\theta} \rightarrow \infty$, the solutions approach the limit plastic ones. The curve for $b / a=1$ is the well-known solution for a circular shaft.

\section{Sokolovsky oval}

The exact solution given by Sokolovsky for an oval cross-section $D$ with major and minor semiaxes $A$ and $B$ and an elliptical elastic core $D_{e}=\left\{(x, y):(x / a)^{2}+(y / b)^{2} \leqslant 1\right\}$ is given by (see [1])

$$
\begin{array}{ll}
\bar{\phi}(x, y)=-\frac{\bar{\theta} a}{a+b}\left(x^{2}+y^{2}\right) & \text { in } D_{e}, \\
\partial \bar{\phi} / \partial y=-\sin \psi, \quad \partial \bar{\phi} / \partial x=-\cos \psi & \text { in } D-D_{e},
\end{array}
$$

where $\psi$ is a parameter related to a point on the elastic-plastic interface. The solution is constructed inversely from an initial elliptical elastic core $D_{e}$ to produce the shape of the oval. The solution is valid only if $A \leqslant 2 B$.

Although the solution is exact, integration of $\phi(x, y)$ over the oval domain needs to be carried out numerically. The restriction $A \leqslant 2 B$ is quite artificial. It only states that no elastoplastic solution with an elliptical core exists when $A>2 B$. There exists a solution in another form.

The solutions for two ovals $(B / A=0.6, B / A=0.4)$ are computed by the present algorithm. The Sokolovsky solution is accurately reproduced as a part of the complete elastoplastic numerical solution for $B / A=0.6$. For $B / A=0.4$ the Sokolovsky method does not apply. But the solution exists and is shown in fig. 3 . 


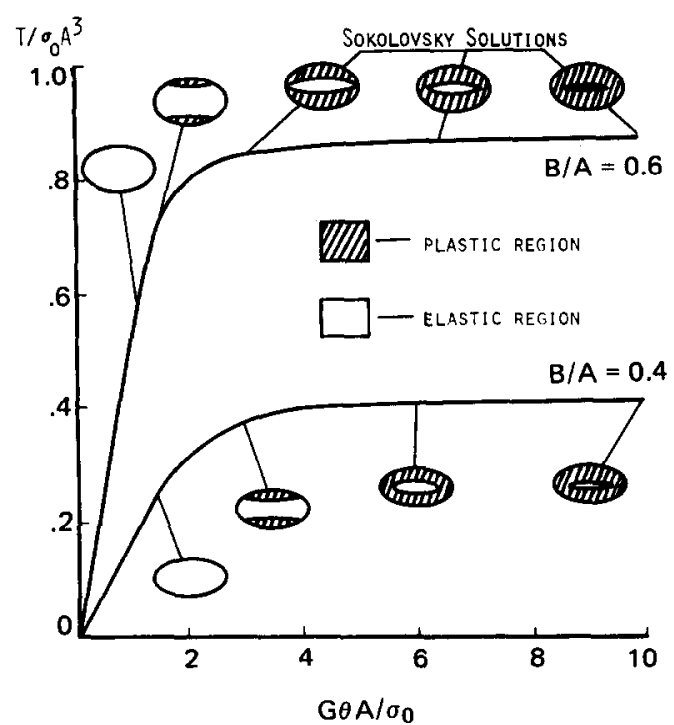

Fig. 3. Torque-angle of twist curves of Sokolovsky oval shafts.

\section{Discussion and conclusions}

The mesh chosen for the quarter-domains has 22 steepest descent lines and 20 unknowns on each line. The algebraic systems have approximately 400 variables. By the standards of most elastoplastic codes these are large problems. However, the computing time for each curve in figs. 2 and 3 is only 23 seconds CPU time on an Amdahl 470. More detailed information on the performance of the conjugate gradient algorithm is given in [9]. We conclude that the use of this algorithm in conjunction with the special mesh technique discussed in this paper presents a more efficient procedure for elastoplastic analysis compared with earlier work [15].

The finite element approximation used in this paper has $\mathrm{O}\left(h^{2}\right)$ error [7], where $h$ is the largest mesh size. For the mesh density chosen the solution $\phi$ and the torque are accurate to three digits. The tolerance, $\epsilon$ on the components of the gradient vector is taken to be $10^{-5}$.

As discussed earlier, the change of $\bar{\theta}$ at each step depends on the amount of information sought. The most economical way is to change $\bar{\theta}$ by an amount that does not require excessive iterations to reach the next solution. This can be achieved by monitoring the number of inner and outer iterations as well as the slope of the torque vs. angle of twist curve at each step during computation.

One of the interesting and important features of elastoplastic analysis is its ability to predict states of residue stresses after a local or global unloading [16]. Although not presented, the method in this paper can be extended to include this aspect and will be presented in a future article.

Plasticity is a field in which the types of nonlinearities lend themselves naturally to computational approaches. Modern numerical methods seem to be the only way to unlock many unsolved problems in the field. 


\section{Acknowledgment}

This work was supported in part by the National Science Foundation Grants MCS-76-06595 and Eng. $-75-17489$.

\section{References}

[1] R. Hill, The mathematical theory of plasticity (Clarendon Press, Oxford, 1950).

[2] W. Prager and P.G. Hodge Jr., Theory of perfectly plastic solids (Wiley, 1951).

[3] A. Mendelson, Plasticity: theory and application (MacMillan, 1968).

[4] J.H. Argyris and A.S.L. Chan, Static and dynamic elastoplastic analysis by the method of finite elements in space and time, International Symposium on Foundations of Plasticity, Warsaw, 1972.

[5] K.-J. Bathe, E. Wilson and F.E. Peterson, SAP4, A Structural Analysis Program for Static and Dynamic Response of Linear System, NSE Report No. EERC 73-11, University of California, Berkeley, Apr. 1974.

[6] J.R. Bunch and D.J. Rose, Sparse matrix computations (Academic Press, 1976).

[7] G. Strang and G.J. Fix, An analysis of the finite element method (Prentice Hall, Englewood Cliffs, NJ, 1973).

[8] D.G. Luenberger, Introduction to linear and nonlinear programming (Addison-Wesley, 1973).

[9] D.P. O'Leary, A gencralized conjugate gradient algorithm for solving a class of quadratic programming problems, STAN-CS77-638 (Stanford Univ. Comp. Sci. Dept., 1977).

[10] R.W. Cottle and M.S. Goheen, A special class of large quadratic programs, Report SOL 76-7 (Stanford Univ. Op. Res. Lab. 1976).

[11] S. Timoshenko and J.N. Goodier, Theory of elasticity, 3rd. ed. (McGraw-Hill, 1970).

[12] W.H. Yang, A practical method for limit torsion problems, Report AMES-19-78 (Univ. Michigan Dept. AMES, 1978).

[13] J. Cea and R. Glowinski, Sur des méthodes d'optimisation par relaxation (R.A.I.R.O. R-3, 1953).

[14] B.T. Polyak, The conjugate gradient method in extermal problems, U.S.S.R. Comp. Math. Math. Phys. 9 (1969).

[15] G. Maier, Mathematical programming methods in structural analysis, Proceedings International Conference on Variational Methods in Engineering II, 8 (Southampton Univ. Press, 1973).

[16] C.T. Herakovich and P.G. Hodge, Elastoplastic torsion of hollow bars by quadratic programming, Int. J. Mech. Sci. 11 (1969) 53-63 